Musées, Patrimoine et Culture scientifiques et techniques

$113 \mid 2007$

septembre - octobre 2007

\title{
Quand les enfants « font » de la Préhistoire
}

\section{Delphine Tuzi}

URL : http://journals.openedition.org/ocim/723

DOI : $10.4000 /$ ocim.723

ISSN : 2108-646X

\section{Éditeur}

OCIM

Édition imprimée

Date de publication : 1 septembre 2007

Pagination : 26-33

ISSN : 0994-1908

Référence électronique

Delphine Tuzi, «Quand les enfants « font » de la Préhistoire », La Lettre de I'OCIM [En ligne], 113 | 2007, mis en ligne le 09 février 2011, consulté le 19 avril 2019. URL : http://journals.openedition.org/ ocim/723 ; DOl : 10.4000/ocim.723 


\section{Quand les enfants " font " de la Préhistoire}

\section{Delphine Tuzi *}

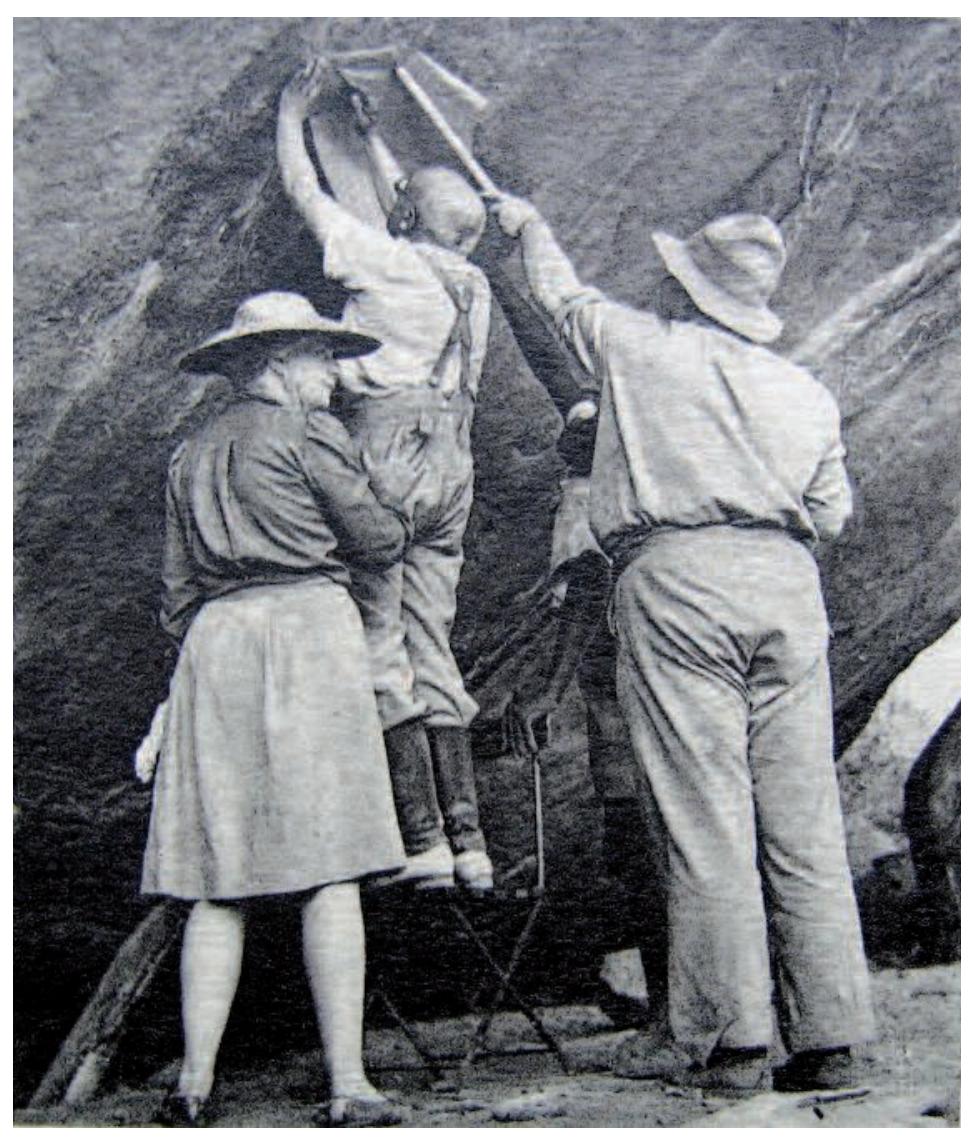

L'abbé Breuil relevant sur calque une œuvre rupestre. C) musée de la Préhistoire, Nemours

* Delphine Tuzi est médiatrice du patrimoine au musée départemental de Préhistoire d'Île-de-France 48 avenue Étienne Dailly 77140 Nemours téléphone + 33164785480 mpif@cg77.fr
La médiation en direction du jeune public organisée à l'occasion d'une exposition temporaire consacrée aux travaux menés par l'abbé Breuil permet aux enfants de découvrir et de mettre en pratique les gestes des hommes de la Préhistoire : les outils mis en place à cette occasion sont ici perçus comme un moyen de fidéliser ce public spécifique.

L'exposition temporaire Sur les chemins de la Préhistoire, l'abbé Breuil, du Périgord à l'Afrique du Sud, reconnue d'intérêt national par le ministère de la Culture et de la Communication, a été organisée par le musée d'Art et d'Histoire Louis Senlecq à l'Isle-Adam et le musée départemental de Préhistoire d'Île-de-France à Nemours avec le concours du service archéologique du Val d'Oise et du CNRS («Action Concertée Incitative Archives Breuil »). Elle a bénéficié de l'appui scientifique de l'Institut de Paléontologie Humaine du Muséum national d'Histoire naturelle, du musée national d'Archéologie de Saint-Germain-en-Laye, ainsi que de nombreux prêts de collections privées et publiques de France.

Ainsi pendant trois mois à Nemours (du 14 octobre 2006 au 14 janvier 2007) le public a pu découvrir la vie et l'œuvre de l'abbé Henri Breuil (1877-1961), inséparables de l'histoire de la Préhistoire. En effet, homme de terrain, il a exploré de nombreux sites préhistoriques et dressé les grandes lignes de la chronologie encore utilisée de nos jours, étudié et relevé de nombreuses œuvres pariétales des grottes ornées de France et d'Afrique du Sud. Il a joué un rôle capital dans la reconnaissance de l'homme préhistorique 
malgré les réticences de l'Église. Il a été le premier grand spécialiste international de l'art préhistorique qu'il a également contribué à faire reconnaître au début du XXe siècle auprès de la communauté scientifique. L'exposition présentait ses travaux (croquis, dessins, relevés aquarellés des œuvres pariétales), les outils témoins de son travail de préhistorien en typologie et en chronologie du Paléolithique ainsi que des œuvres d'art mobilier préhistorique sur os ou sur pierre. Elle se terminait par une visite virtuelle de la grotte de Lascaux en 3D, création audiovisuelle originale du Centre national de Préhistoire de Périgueux, intitulée Sur les pas de Brevil.

Dans la plupart des musées, les expositions temporaires ne sont pas réalisées pour les enfants. Elles appartiennent au monde du savoir et des adultes. Il faut donc les aborder d'une manière spécifique avec de jeunes publics afin de donner à ceux-ci l'envie de fréquenter les musées, et d'une façon générale, d'aiguiser leur curiosité pour les collections d'objets.

Pour rendre ses expositions accessibles au jeune public, le musée départemental de Préhistoire d'Îlede-France à Nemours met régulièrement en place, à l'occasion des expositions temporaires, des outils pédagogiques spécifiques : questionnaires, livrets d'aide à la visite, animations. Pour l'exposition Sur les chemins de la Préhistoire, l'abbé Breuil, du Périgord à l'Afrique du Sud, le service des publics a développé des actions permettant d'adapter celle-ci à un jeune public, par la création de livrets d'aide à la visite, pour le public individuel, et la mise en place d'une animation pour les scolaires.

\section{Médiation de l'exposition pour le jeune public individuel}

Pour l'exposition précédente Temps du Rêve, Temps des Hommes, le service des publics avait créé un livret pour les 7-13 ans intitulé Temps du Rêve, Temps des enfants. Pour celle-ci, la volonté a été de réaliser deux livrets adaptés à des tranches d'âges différentes : Dis l'abbé, raconte-moi la Préhistoire pour les enfants de 7 à 9 ans et Pas à pas avec l'abbé Brenil pour les plus de 10 ans. Distribués gratuitement à l'accueil du musée, ils permettaient aux enfants venus en famille de découvrir l'exposition d'une façon ludique, au moyen d'enquêtes ou de jeux .

\section{Les objectifs}

En réalisant ces livrets, l'équipe de médiation cherchait à répondre aux objectifs suivants :

- rendre l'exposition attractive pour les enfants,
- montrer à travers la vie de l'abbé Breuil le travail du préhistorien,

- faire comprendre l'intérêt des relevés archéologiques, particulièrement ceux réalisés par l'abbé Breuil (quelquefois ce sont les seuls témoignages qu'il reste des œuvres préhistoriques),

- montrer l'intérêt d'étudier les objets préhistoriques.

\section{Les contraintes}

Lors de la mise en place d'un tel outil, l'objectif est bien entendu de le mettre à disposition des visiteurs dès l'ouverture au public de l'exposition. Cela nécessite qu'une grande partie de sa conception se fasse en amont du montage de l'exposition. Or, le plan comme le contenu du livret sont intimement liés au cheminement et à la scénographie de l'exposition. De plus, la réalisation d'un livret implique généralement la collaboration de plusieurs services ou prestataires qui ont leurs propres procédures, contraintes et objectifs. L'exercice s'apparente donc souvent à un parcours d'équilibriste, avec d'un côté le risque de concevoir un outil passe-partout déconnecté du discours de l'exposition et de l'autre le risque d'un retard de livraison.

\section{La réalisation des livrets}

Pour l'exposition Sur les chemins de la Préhistoire, l'abbé Breuil, du Périgord à l'Afrique du Sud, nous avons opté pour une réalisation des livrets en interne au Conseil général de Seine-et-Marne. Il s'agissait donc d'une collaboration entre services d'une même collectivité, à savoir le musée de Préhistoire, la sous direction des musées, la direction de la communication et l'imprimerie départementale.

Par ailleurs, l'exposition avait été auparavant présentée au musée Louis Senlecq de L'Isle-Adam. Cela a permis au service des publics de s'appuyer sur les collections et les documents présentés là-bas, pour commencer très tôt la conception des livrets. En sachant néanmoins quà Nemours, pour des raisons d'espace et de conservation préventive, ni le cheminement ni les relevés ne seraient les mêmes.

L'anticipation du service des publics et l'implication des différents services du Conseil général de Seineet-Marne ont permis de tenir les délais tout en assurant aux outils de médiation une qualité en terme de contenu et de graphisme. Ainsi 600 livrets ont été imprimés pour le jour de l'inauguration.

\section{Description des livrets}

Les deux livrets, d'une dizaine de pages chacun, ont été conçus d'après les thèmes privilégiés par l'exposition : les voyages de l'abbé Breuil, l'étude de l'art 


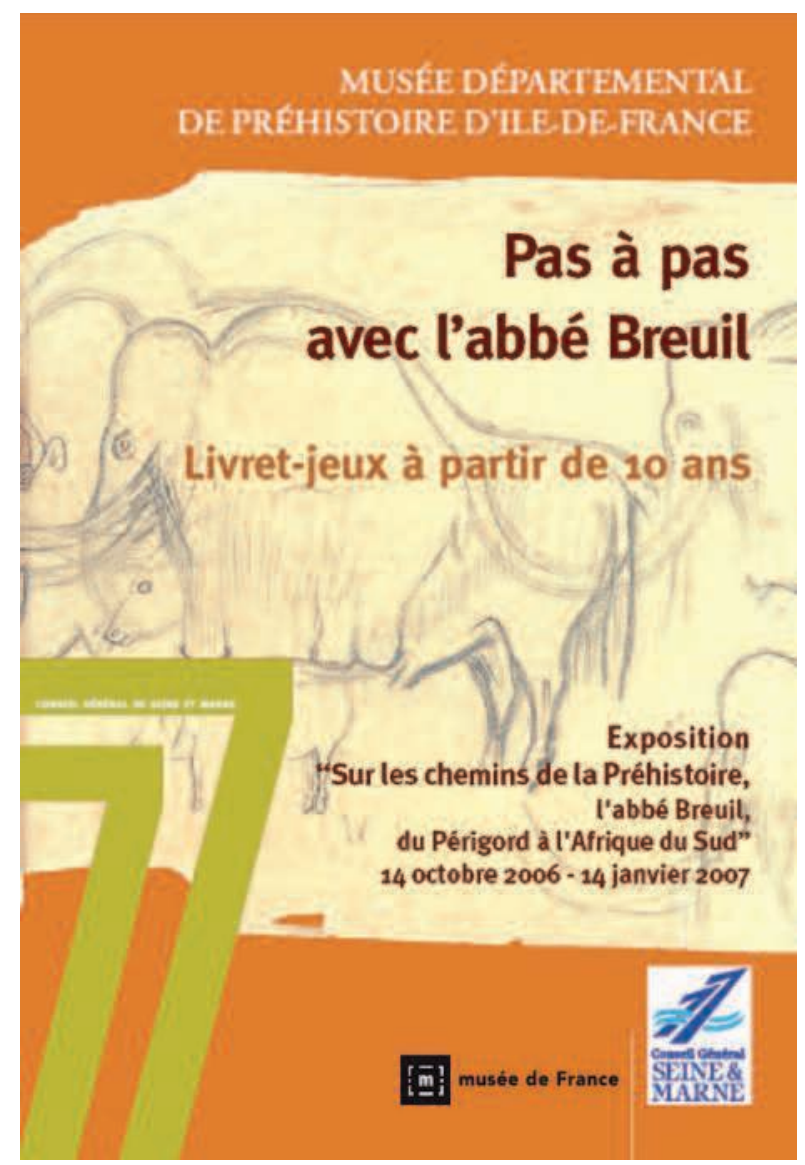

pariétal (travail de relevé), l'étude de l'art mobilier, le rôle de l'étude des outils préhistoriques dans la définition d'une chronologie. En observant les objets, en lisant certains drops ou certaines notices, en reproduisant les œuvres, les enfants recherchaient dans l'exposition les éléments permettant la réalisation des activités-jeux du livret. Avant chaque jeu, un court texte explicatif et des consignes étaient donnés à l'enfant sur les différentes thématiques. À l'issue de la visite, les enfants se voyaient remettre les solutions de tous les jeux de leur livret.

\section{Médiation de l'exposition pour un public scolaire}

Depuis une dizaine d'années, le musée a fait l'effort d'adapter les expositions temporaires aux jeunes enfants. Cependant par manque de personnel, le service des publics ne pouvait pas proposer systématiquement une animation pour les scolaires en lien avec les expositions. Depuis 2005, le recrutement ponctuel d'un médiateur a permis de développer de tels outils. Ainsi pour l'exposition Temps

\section{L'abbé Breuil interprète ses découvertes}

Souviens-toi de la gravure-peinture de la grotte des Trois-Frères (page 5). L'abbé Breuil a cru voir un sorcier ou un dieu cornu dans cette représentation.

En fait, en dehors des animaux les plus réatistes, il est souvent bien difficile de savoir ce que les hommes préhistoriques ont voulu représenter.

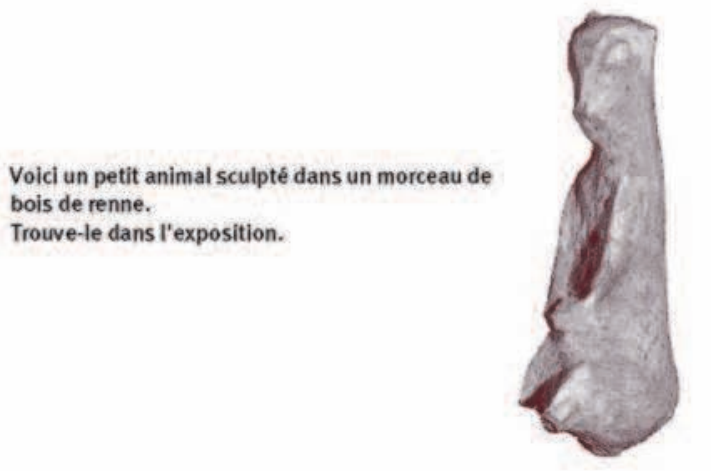

Les archéologues hésitent sur l'identité de cet animal.

A ton avis, de quel animal s'agit-il?

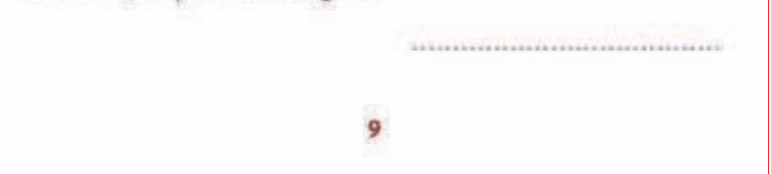

Le livret-jeu à destination des enfants de + de 10 ans

(c) musée de la Préhistoire, Nemours

du Rêve, Temps des hommes, une activité de création artistique autour de l'art aborigène avait été proposée aux écoles.

À l'occasion de l'exposition Sur les chemins de la Préhistoire, l'abbé Breuil, du Périgord à l'Afrique du Sud, l'équipe de médiation a conçu une animation particulière basée sur les techniques de relevés d'œuvres pariétales. Ce thème a permis d'aborder l'art préhistorique d'une manière originale. Cependant sa mise en place ne s'est pas faite sans difficultés notamment pour la création d'outils pédagogiques adaptés.

Les objectifs de l'animation pour les scolaires L'objectif principal était de faire comprendre l'intérêt des relevés archéologiques des gravures et peintures préhistoriques : ils constituent la base de la recherche sur l'art pariétal, une lecture et une vision synthétique de l'œuvre. En ce qui concerne les relevés de l'abbé Breuil, pour certains ils représentent les seules traces de ces peintures : par exemple, on peut grâce à eux connaître et étudier certaines peintures aujourd'hui disparues de la grotte de Bédeillac en Ariège. 
Un autre objectif de l'animation était d'appliquer les techniques de relevés archéologiques d'œuvres pariétales utilisées par les préhistoriens : comment passer d'une peinture ou d'une gravure sur un support en trois dimensions à un relevé papier ? L'abbé Breuil réalisait une copie par décalque posée directement sur les œuvres réalisées sur des parois non friables.

Aujourd'hui, les relevés se font sur film transparent tendu sur des barreaux d'aluminium à partir de bâtis d'échafaudage montés selon la configuration de la paroi. Le film approche de très près l'œuvre mais ne la touche pas. Une autre technique de plus en plus employée est la photogrammétrie : il s'agit d'un relevé tridimensionnel dont le résultat final donne une maquette virtuelle de la paroi. Il est réalisé à l'aide de prises de vues photographiques stéréoscopiques et de mesures topographiques.

Enfin tout au long de l'animation il s'agissait de montrer que l'Archéologie est une discipline collective : le relevé d'une figure ou d'un groupe de figures prend tout son sens à partir du moment où il est associé aux autres relevés de la paroi, restituant ainsi l'œuvre en entier.

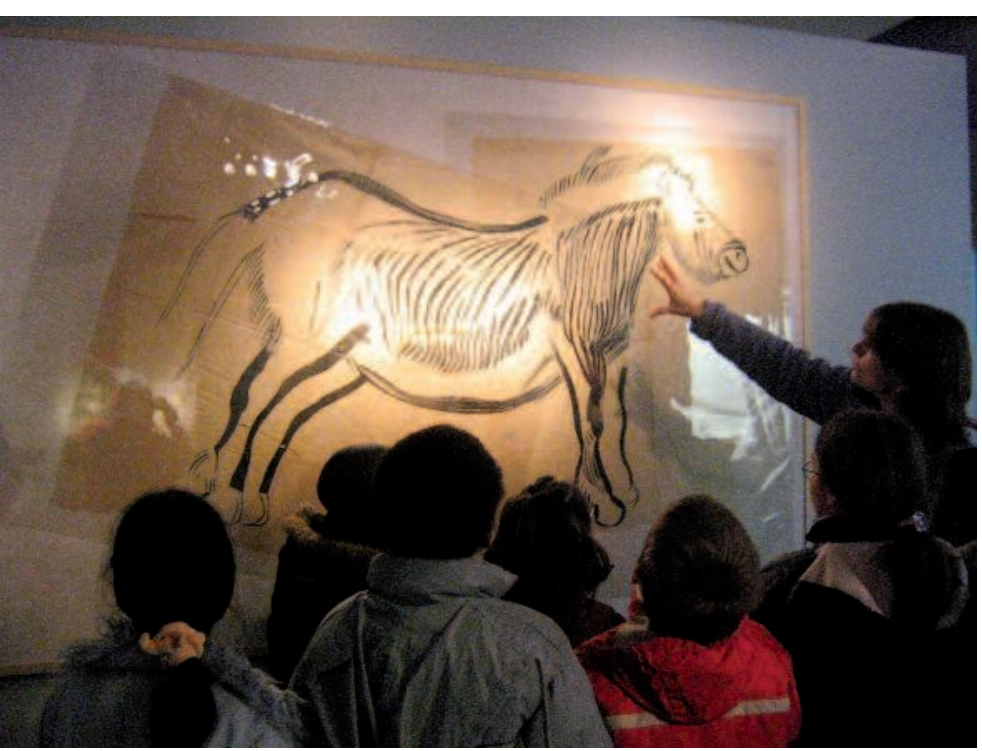

Lecture avec les enfants d'un relevé de l'abbé Breuil dans l'exposition: Cheval de la grotte de Niaux en Ariège. () musée de la Préhistoire, Nemours

\section{Les contraintes imposées}

La salle du musée dédiée aux expositions temporaires est d'une superficie relativement modeste (environ $150 \mathrm{~m}^{2}$ ) : la circulation de groupes importants, comme une classe entière de 30 enfants, n'y est donc pas vraiment envisageable.
Une autre contrainte récurrente liée à la structure, est le manque d'espace d'animation. Le musée possède deux circuits de visite de quatre salles chacun : un circuit court destiné au grand public et un circuit long conseillé aux visiteurs soucieux d'approfondir leurs connaissances dans des salles supplémentaires. Les animations proposées dans le musée sont alors réalisées dans les salles du circuit long, nommées salles «bis». Le plus souvent deux salles «bis » d'exposition permanente sont occupées en même temps toute la journée par ces activités. Il était donc impossible d'ajouter une troisième animation dans une autre salle.

Des contraintes liées également aux groupes scolaires se sont imposées. En effet les classes sont tributaires d'horaires imposés soit par leur transport (privé ou public), soit par leur établissement. Ce qui leur laisse parfois un temps réduit de présence au musée.

Enfin le personnel du service des publics ne possédait pas suffisamment de temps et de moyens artistiques pour créer les outils spécifiques nécessaires à cette animation.

\section{La mise en place de l'animation}

Pour résoudre le problème de l'exiguïté des locaux de l'exposition et des espaces d'animation, il a donc été décidé de partager la classe en deux : pendant qu'un demi-groupe participait à la visite de l'exposition et à l'animation avec un médiateur dans le hall d'entrée du musée, l'autre groupe visitait avec l'enseignant l'exposition permanente à l'aide d'un dossier pédagogique fourni par le musée. Puis les groupes étaient inversés ; pour participer à cette animation, la classe devait donc rester la journée entière au musée.

Pour répondre aux contraintes horaires des classes, l'animation a été organisée de façon à ce que la durée corresponde au temps minimum passé généralement au musée par les élèves (environ 1 h 30 par demi-journée).

Enfin pour faire travailler les enfants sur les techniques de relevé des œuvres pariétales, il fallait créer un support que le service des publics ne pouvait réaliser par manque de temps et d'aptitude technique. Le musée a donc fait appel à Michel Grenet, préhistorien plasticien, pour travailler à la création d'outils adaptés (1). À la demande du service des publics, il a créé deux fac-similés de parois de grotte de 120 X $250 \mathrm{~cm}$ chacune : l'une ornée de gravures et l'autre de peintures, inspirées d'œuvres de différentes grottes ornées du Paléolithique comme 


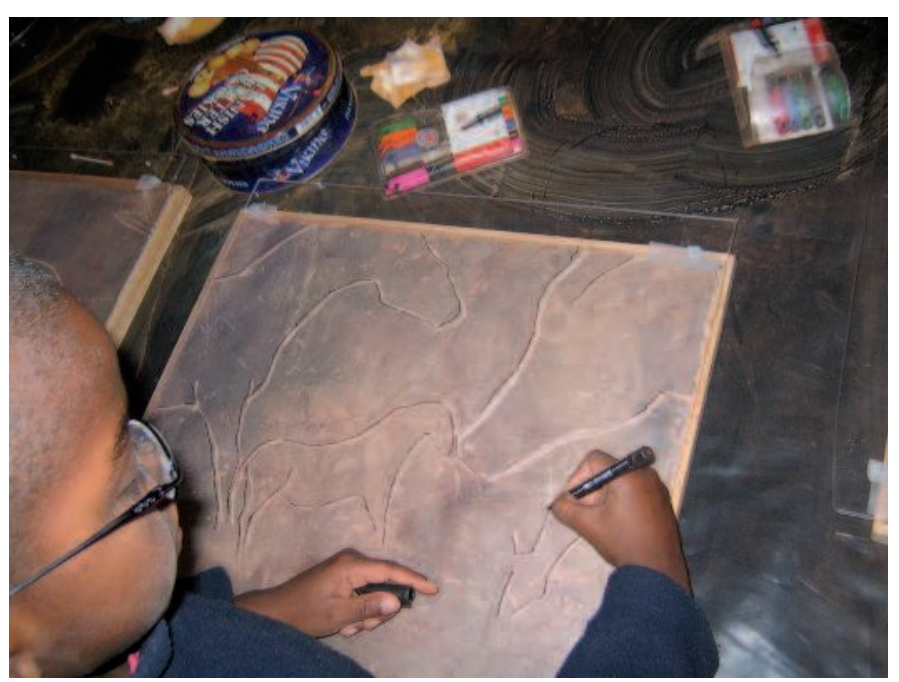

Un enfant décalque les contours d'une gravure sur un film transparent. () musée de la Préhistoire, Nemours

Lascaux, Cosquer, Chauvet, Les Combarelles, Font de Gaume, Rouffignac. Réalisées en placoplâtre, avec des enduits spécifiques et du plâtre polyester, les parois présentent un aspect " surface naturelle de grotte ». Elles ont été ensuite protégées de résine acrylique ou vinyle d'aspect satin ou mat. Enfin, elles ont chacune été découpées en quinze panneaux de $40 \times 50 \mathrm{~cm}$, protégées par un cadre en bois, de manière à ce que chaque enfant puisse disposer d'un panneau. Ainsi la classe entière aura abordé, dans la journée, les différentes techniques utilisées dans l'art pariétal : la gravure pour un groupe, la peinture pour l'autre.

\section{Déroulement de l'animation}

L'animation se décomposait en quatre temps : la visite de l'exposition, l'activité autour du relevé archéologique, puis la restitution de l'ensemble de la paroi et enfin la découverte d'une technique plus moderne de relevé grâce à une séance vidéo.

\section{Visite de l'exposition}

La présentation de l'exposition par l'animateur s'organisait autour des quatre thèmes phares de l'exposition, lesquels avaient déjà constitué la trame des livrets : les voyages de l'abbé Breuil, le rôle de l'étude d'outils préhistoriques dans la définition d'une chronologie des temps préhistoriques, l'étude de l'art mobilier, l'étude de l'art pariétal (observation des relevés de l'abbé Breuil).

\section{L'animation}

Au cours de l'animation, les enfants s'initiaient aux gestes du préhistorien en réalisant le relevé d'une

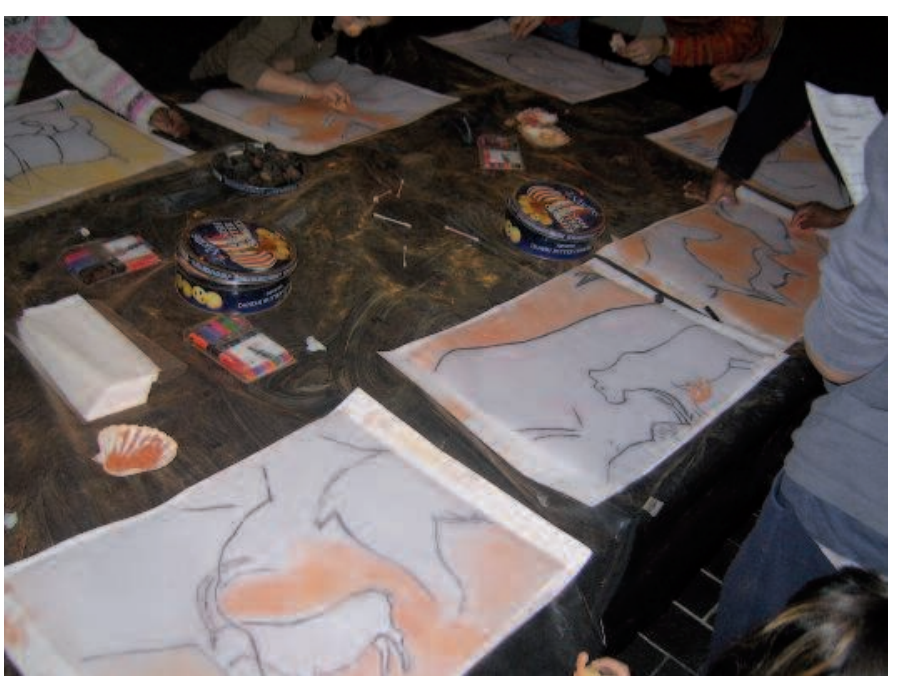

Mise en couleur des relevés des gravures (c) musée de la Préhistoire, Nemours

œuvre pariétale. Chaque enfant disposait d'un panneau représentant un fragment de la paroi peinte ou gravée. Le médiateur leur laissait le temps d'observer et de toucher les reliefs de la fausse paroi. L'animation se décomposait en trois ou quatre étapes :

- première étape : décalquer les contours de la peinture ou de la gravure. Pour ce faire, le médiateur déposait sur le panneau, une plaque de plexiglas sur laquelle les enfants disposaient ensuite une feuille plastique souple transparente. Ils décalquaient ainsi les contours observés sur ce plastique à l'aide de feutres indélébiles.

- deuxième étape : transposition du relevé plastique sur papier calque. En posant un calque sur le film transparent, les élèves dessinaient les contours des gravures ou des peintures à l'aide de charbon de bois (pour les gravures ou les contours de couleur noire) et de craies d'ocre rouge ou jaune (pour les peintures).

- troisième étape : mise en couleur du relevé. À l'aide de poudre d'ocre et de charbon de bois les enfants pouvaient restituer fidèlement sur le papier calque les couleurs des œuvres peintes ou bien les couleurs naturelles de la paroi pour les œuvres gravées.

- quatrième étape : description écrite. Si le temps le permettait les enfants complétaient enfin une fiche de description précisant les couleurs utilisées, les techniques employées et les sujets représentés dans l'œuvre étudiée.

\section{La restitution}

À l'issue de l'animation, une fois tous les relevés réalisés, le médiateur faisait défiler sur un écran à l'aide d'un diaporama les photographies de toutes les 
plaques pour restituer l'ensemble de la paroi : les enfants positionnaient alors leur relevé au bon endroit et constituaient ainsi pièce par pièce le relevé de l'ensemble de la paroi.

\section{Découverte d'une technique récente de relevé : la photogrammétrie}

L'animation se terminait par le visionnage du film en 3D Sur les pas de Breuil consacré à la grotte de Lascaux, commandé et réalisé à l'occasion de cette exposition. Ce film permettait de montrer aux enfants une grotte aujourd'hui fermée au public pour des raisons de conservation. Il mettait également en évidence une technique moderne de relevé (la photogrammétrie) et les nombreuses possibilités qu'elle apporte pour la recherche archéologique.

\section{Le bilan des outils de médiation mis en place}

\section{En terme de public}

\section{Les livrets}

Les enseignants, ou les individuels ayant observé l'utilisation du livret par les enfants, nous ont fait part d'observations positives quant à la qualité du contenu et des illustrations. Ils nous ont aussi indiqué la difficulté de certains enfants pour trouver les réponses dans l'exposition, surtout sur le jeu dont le sujet était la stratigraphie et la chronologie des outils. Sur les 600 livrets imprimés, 300 étaient destinés aux enfants de 7-9 ans et 300 pour les enfants à partir de 10 ans. La distribution du livret Dis l'abbé, raconte-moi la Préhistoire pour les 7-9 ans a été plus importante que celle du livret Pas à pas avec l'abbé Breuil destiné aux plus de 10 ans. Cela semble correspondre à la tranche d'âge la plus couramment accueillie au musée en ce qui concerne le jeune public.

\section{L'animation}

Sur une courte période de trois mois 26 ateliers ont été réalisés en plus des ateliers proposés habituellement. Cela correspond à la participation de 337 enfants sur les 17000 accueillis au musée dans l'année. C'est un résultat à peu près comparable à celui obtenu lors de l'exposition précédente Temps du rêve, Temps des Hommes. Il s'agit à $90 \%$ d'enfants de classe de CE2 et à $10 \%$ de collégiens (des $6^{\mathrm{e}}$ notamment), ce qui correspond à la fréquentation habituelle du musée. Ce sont des élèves venus principalement de Seine-et-Marne $(51 \%)$ et d'Essonne (36\%).

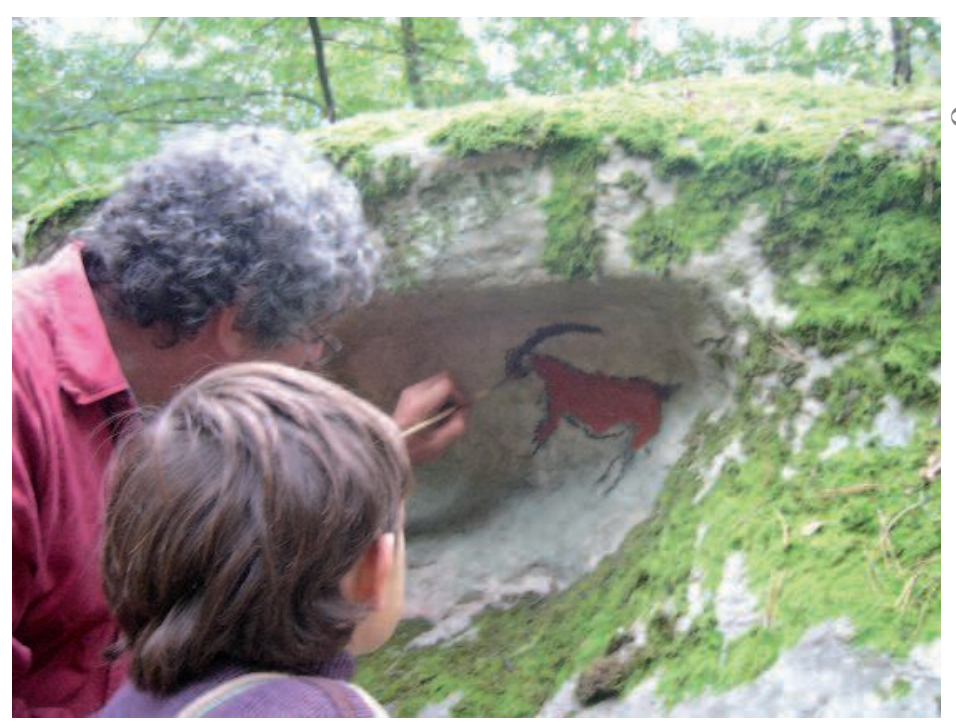

Rochers du parc du musée ornés par Michel Grenet à l'occasion de sa médiation pour la Fête de la Science.

(c) musée de la Préhistoire, Nemours

L'animation a pu également être proposée lors de la Fête de la Science et du stage de la Toussaint à destination des collégiens de Nemours :

- dans le cadre de la $15^{\mathrm{e}}$ édition de la Fête de la Science, le musée a invité le public à découvrir les techniques et les matériaux de l'art pariétal grâce à l'intervention de Michel Grenet. Alternant discours, démonstrations et participation du public, il a évoqué les gestes artistiques de nos ancêtres sur les parois de blocs de grès présents dans le parc du musée. À l'occasion de cette journée, le service des publics a proposé dans le hall du musée l'animation prévue pour les scolaires. Ce fut l'occasion de tester différents points de l'animation scolaire à venir, comme les techniques choisies en matière de mise en couleurs.

- depuis plusieurs années le musée propose pendant les vacances de la Toussaint un mini stage aux collégiens de Nemours. Celui-ci s'inscrit dans un partenariat avec la ville de Nemours dans le cadre de la "Caravane des cultures », opération présentant les activités culturelles des associations et services publics de la ville aux collégiens de Nemours. Cette année, en raison de l'exposition, le service des publics a axé le stage sur le thème de l'art paléolithique : du travail de relevé à l'expérimentation de gestes vieux de 30000 ans. Ce stage a également été l'occasion de tester une première fois l'exposition avec des collégiens. 


\section{Bilan en terme d'objectifs}

Pour différentes raisons, il n'a pas été fait d'évaluation précise de ces outils de médiation. Néanmoins, un certain nombre d'observations peuvent faire état de bilan qualitatif.

\section{Pour les livrets}

L'objectif de départ, rendre plus attractive l'exposition, semble avoir bien été rempli. En effet, à l'aide de leurs livrets les enfants ont circulé en autonomie dans l'exposition et ont pu, grâce aux jeux, porter un regard différent sur les collections : observations d'objets exceptionnels comme les œuvres d'art mobilier et compréhension de l'importance des relevés de l'abbé Breuil...

\section{Pour l'animation}

Du point de vue de l'Éducation nationale, l'animation répondait à plusieurs objectifs inscrits dans les programmes scolaires :

- en histoire : aborder la Préhistoire en retraçant la vie de nos ancêtres et leur environnement.

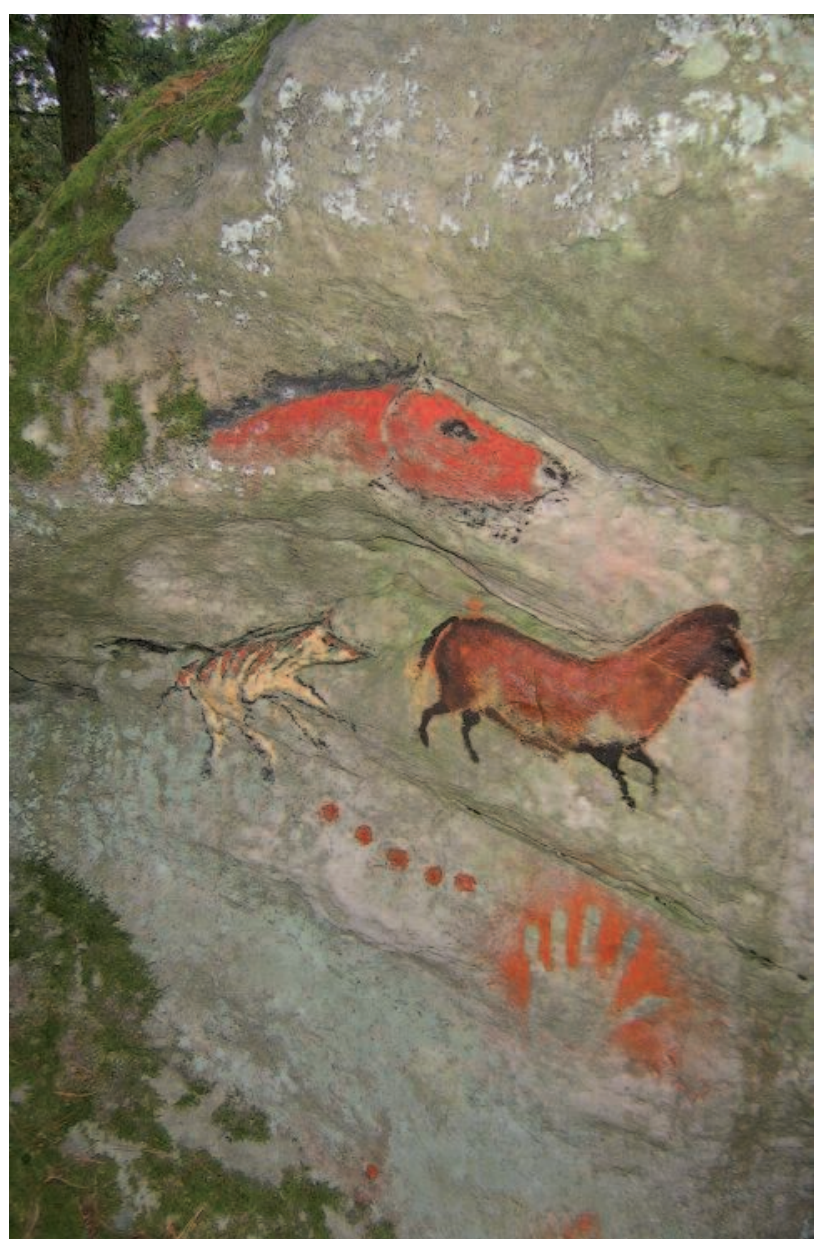

Paroi en grès peinte. (c) musée de la Préhistoire, Nemours
- pour la maîtrise de la langue française : faire un travail écrit autour de la description de l'œuvre qui complète le relevé.

- en éducation artistique, l'animation a permis de découvrir les différentes techniques artistiques utilisées par les hommes préhistoriques (la sculpture, la gravure, la peinture), de manipuler des pigments naturels (ocres, charbon de bois), d'être en contact direct (objets d'art mobilier exposés) ou indirect avec des œuvres préhistoriques (relevés, photographies) et de familiariser les enfants aux différents types d'images (reproduction d'une œuvre pariétale sur papier ou sur plaque, reproduction en 3D en image de synthèse).

- pour la réalisation d'un travail en groupe : dans l'animation l'enfant réalise individuellement un relevé archéologique, mais ce travail s'inscrit plus largement dans une production en classe entière. En effet ce travail individuel ne peut être compréhensible que s'il y a une mise en commun avec les autres enfants, laquelle permet la restitution de la paroi en entier.

Du point de vue du musée, l'animation est une expérience satisfaisante. Elle a permis au service des publics de diversifier les animations proposées habituellement. Elle a également mis en valeur une facette du métier d'archéologue. Souvent oublié, le travail de relevé est indispensable en Archéologie, puisqu'il est la base de l'étude scientifique et une représentation graphique des découvertes.

\section{Perspectives d'avenir}

Ces outils de médiation (animations ou livrets d'aide à la visite d'une exposition), semblent des moyens tout à fait efficaces pour fidéliser les publics et rendre accessibles les expositions à des publics variés. Grâce à la création de livrets, les enfants peuvent désormais s'approprier et découvrir d'une façon ludique des expositions qui ne leur étaient pas forcément destinées. Ainsi pour eux, le musée est un lieu qu'ils vont avoir envie de fréquenter. Dans ce cadre, les agents d'accueil ont un rôle primordial. En effet, ils sont les premiers en contact avec le public. Ils distribuent les livrets à l'accueil et donnent les informations aux visiteurs. Il est donc très important d'informer le personnel d'accueil sur le contenu de l'exposition, celui des livrets et les modalités de distribution et d'utilisation de ceux-ci.

Quant à l'animation, elle a permis de créer un partenariat privilégié avec les écoles locales. À chaque exposition temporaire du musée, celles-ci n’hésitent plus désormais à participer aux nouvelles animations 
en relation avec l'exposition, même si le thème de celle-ci ne semble pas en rapport direct avec le programme scolaire. L'animation a aussi permis au musée d'acquérir des outils pédagogiques d'une grande qualité : les fac-similés de paroi réalisés par Michel Grenet. Ceux-ci présentent différents niveaux de lecture, certains d'ailleurs non exploités dans l'animation. Ils le seront probablement lors d'animations ultérieures.

Cependant, la mise en place de différents outils nécessite que l'équipe de médiation soit systématiquement intégrée au projet muséographique des expositions. Elle peut effectivement apporter un regard différent sur la scénographie et les textes. Il est impératif qu'elle puisse avoir accès à toutes les informations concernant l'exposition lors de la préparation des livrets et des animations. Sans ces outils de médiation, l'exposition temporaire aurait probablement rencontré un succès moindre auprès du jeune public.

\section{Note}

(1) Rattaché à l'unité du CNRS UMR 6130, au centre d'Étude en Préhistoire et Archéologie médiévale de Sophia-Antipolis, Michel Grenet collabore depuis de nombreuses années avec des équipes de chercheurs lors de programmes ou de missions en France et à l'étranger. Affilié à la maison des Artistes, il réalise depuis bientôt vingt ans des illustrations pour la presse ou à destination d'ouvrages spécialisés et d'expositions muséographiques. Médiateur en Préhistoire, il encadre depuis une douzaine d'années des stages de formation et présente des animations sur le thème de la Préhistoire. Il poursuit parallèlement ses activités de plasticien et expose dans plusieurs galeries en France et en Europe du Nord. 